\title{
RePos: Relative Position Estimation of UHF-RFID Tags for Item-level Localization
}

\author{
Chenglong $\mathrm{Li}^{1 \dagger}{ }^{1 \dagger}$ Emmeric Tanghe ${ }^{1}$, David Plets ${ }^{1}$, Pieter Suanet ${ }^{2}$, Jeroen Hoebeke ${ }^{3}$, \\ Eli De Poorter ${ }^{3}$, Wout Joseph ${ }^{1}$ \\ ${ }^{1}$ INTEC-WAVES, Ghent University-IMEC, Ghent, Belgium \\ 2 Aucxis cvba, Stekene, Belgium \\ ${ }^{3}$ INTEC-IDLab, Ghent University-IMEC, Ghent, Belgium \\ ${ }^{\dagger}$ Email: chenglong.li@ugent.be
}

\begin{abstract}
Radio frequency identification (RFID) technology brings tremendous applications in location-based services. Specifically, ultra-high frequency (UHF) RFID tag positioning based on phase (difference) of arrival (PoA/PDoA) has won great attention, due to its better positioning accuracy than signal strength-based methods. In most cases, such as logistics, retailing, and smart inventory management, the relative orders of the objects are much more attractive than absolute positions with centimeterlevel accuracy. In this paper, a relative positioning (RePos) approach based on inter-tag distance and direction estimation is proposed. In the RePos positioning system, the measured phases are reconstructed based on unwrapping method. Then the distances from antenna to the tags are calculated using the distance differences of pairs of antenna's positions via a leastsquares method. The relative relationships of the tags, including relative distances and angles, are obtained based on the geometry information extracted from PDoA. The experimental results show that the RePos RFID positioning system can realize about 0.28 meter ranging accuracy, and distinguish the levels and columns without ambiguity.
\end{abstract}

\section{INTRODUCTION}

Radio frequency identification (RFID) technology has attracted great interest from both industry and research community due to low cost and easy deployment. Besides the tagsticking object identification, the potential in fine-grained location services provides new solutions to asset management and tracking. For example, for library management with millions of collect books, as well as warehouse management. The staffs always hope to locate track the objects, or find the misplaced ones. Passive ultra-high frequency (UHF) RFID technology provides a flexible way to make the expectations come true.

Most of available solutions adopt received signal strength (RSS)-based, phase (difference) of arrival (PoA/PDoA)-based, and angle of arrival (AoA)-based methods. Among them, PoA/PDoA is popular as a result of its robustness to complex indoor environments. Generally, the localization scheme can be classified as static-reader with static-tags [1], static-reader with moving-tags (e.g. tracking pieces of luggage on conveyor belt [2]-[4]), and moving-reader with static-tags (e.g. item positioning in warehouse [5]). As for the techniques involved, synthetic aperture radar (SAR) method utilizes the concept of virtual spatial diversity. It has been turned out to be a quite promising approach to UHF-RFID tag positioning, due to the absence of burden on changing the hardware configuration, or requiring troublesome hardware deployment (multiple readers/tags). However, for a number of applications, the absolute positions of items with centimeter-level accuracy are not required, but the relative locations, such as for library and inventory management.

In [6], OTrack utilized RSS combined with response reception ratio with a given sliding window to distinguish the order of luggage on the conveyor. STPP was proposed in [7], which ordered the tags horizontally and vertically based on the spatial and temporal phase profiling when antenna moving along the tags. However, sufficient sampling is required for STPP to construct the reliable phase profiling. Based on the SAR method, MobiTagbot [8] achieved much better localization performance than OTrack and STPP, which is hologrambased positioning method intrinsically. Recently, a new method (HMO) to distinguish the tags' order was proposed based on the RSSI and phase changing [1]. But HMO was established based on specific scenario when people moving between the reader antennas and tags, which can not be applied for asset management directly in case of no object going through the antennas and tags.

In this paper, a relative positioning method is proposed. Firstly, the distances between antenna and tags within the interrogation region are estimated based on PDoA using the least-squares method. Then the relative locations including the distances and angles, are obtained based on the geometry. The remainder of this paper is organized as follows. In section II, the measured phase unwrapping method is proposed, then the distance estimation with interference mitigation, and the relative positioning algorithm are presented. Section III gives the system configuration and analyzes experimental results. Section IV concludes this paper.

\section{Tags Relative Positioning Method}

The communication between an UHF-RFID reader and a passive tag depends on the backscatter modulation resulting from the varying load impedance, which consists of the forward and the backscatter link. The forward link powers the passive RFID tags, while the backscatter link reads the message in the tags. Consider that a narrowband UHF-RFID reader co-locates with transmitter and receiver. The phase 
extracted from the baseband signal at the $n$-th observation can be given by

$$
\phi[n]=4 \pi \frac{d[n]}{\lambda}+\underbrace{\varphi_{T, R}+\varphi_{T a g}}_{\varphi_{0}},
$$

where $\lambda$ is the wavelength, $d$ the distance between the tag and antenna, $\varphi_{T, R}$ and $\varphi_{T a g}$ are the phase rotation caused by the transceiver and tag, respectively. Due to the modulo $2 \pi$ operation, the measured phase is denoted as $\phi_{m}[n]=\bmod$ $\left(4 \pi \frac{d[n]}{\lambda}+\varphi_{0}, 2 \pi\right)$, namely,

$$
4 \pi \frac{d[n]}{\lambda}+\varphi_{0}=\phi_{m}[n]+2 k_{n} \pi .
$$

where $2 k_{n} \pi,\left(k_{n} \in \mathbb{K}\right)$ represents the phase ambiguity.

\section{A. Phase Unwrapping}

The modulo- $2 \pi$ operation causes phase ambiguity for each measured phase, which brings challenge to the ranging estimation. But when the measured phase satisfies spatial sampling theory, namely $\frac{4 \pi}{\lambda}|d[n+1]-d[n]|=\frac{4 \pi}{\lambda}\left|\Delta d_{(n+1, n)}\right|<\pi$, we can use the unwrapping algorithm [9] to get the reconstructed unwrapping phase $\psi_{m}[n], n=1,2, \cdots, N-1$,

$$
\left\{\begin{array}{l}
\psi_{m}[1]=\phi_{m}[1] \\
\psi_{m}[n+1]=\phi_{m}[n+1]-2 \pi\left\lfloor\frac{\phi_{m}[n+1]-\psi_{m}[n]}{2 \pi}+\frac{1}{2}\right\rfloor,
\end{array}\right.
$$

where $\lfloor\bullet\rfloor$ denotes the operator rounding toward negative infinity. In the algorithm, the first measured phase is selected as the reference without loss of generality.

Remark 1. After unwrapping, all the reconstructed phases have the same phase ambiguity.

Proof. According to the spatial sampling theory, the phase shift at two adjacent positions should less than $\pi$, so the measured phase differences will satisfy $\left|\phi_{m}[n+1]-\phi_{m}[n]\right|<$ $2 \pi$. We assume that $\psi_{m}[n]+2 k_{n} \pi=\phi_{m}[n]+2 k_{n} \pi$ without loss of generality, so integer difference $\Delta k^{(n+1, n)}=k_{n+1}-k_{n}$ with regards to $\Delta \psi_{m}^{(n+1, n)}=\phi_{m}[n+1]-\psi_{m}[n]$ satisfies

$$
\Delta k^{(n+1, n)}=\left\{\begin{array}{cc}
-1, & \pi<\Delta \phi_{m}^{(n+1, n)}<2 \pi \\
0, & -\pi<\Delta \phi_{m}^{(n+1, n)}<\pi \\
1, & -2 \pi<\Delta \phi_{m}^{(n+1, n)}<-\pi
\end{array} .\right.
$$

So we have

$$
\begin{aligned}
& \phi_{m}[n+1]+2 k_{n+1} \pi \\
= & \psi_{m}[n+1]+2 \pi\left\lfloor\frac{\Delta \phi_{m}^{(n+1, n)}}{2 \pi}+\frac{1}{2}\right\rfloor+2 k_{n+1} \pi \\
= & \psi_{m}[n+1]+2 k_{n+1} \pi+2 \pi\left\{\begin{array}{l}
1, \pi<\Delta \phi_{m}^{(n+1, n)}<2 \pi \\
0,-\pi<\Delta \phi_{m}^{(n+1, n)}<\pi \\
-1,-2 \pi<\Delta \phi_{m}^{(n+1, n)}<-\pi
\end{array}\right. \\
= & \psi_{m}[n+1]+2 k_{n} \pi .
\end{aligned}
$$

So the $(n+1)$-th and $n$-th phases have the same phase ambiguity $2 k_{n} \pi$ after unwrapping. Likewise, any two adjacent phases satisfying the spatial sampling theory have the same phase ambiguity.

However, the criterion of spatial sampling theory may be not easy to meet in practical applications considering the reader's read rate under specific systems setting, antenna's moving velocity for continuous moving scenario, channel fading, and measurement errors, etc. In this paper, we develop an iterative phase unwrapping algorithm to further relieve the spatial sampling theory's phase interval to $2 \pi$ instead of $\pi$ in traditional method, namely, $\frac{4 \pi}{\lambda}\left|\Delta d_{(n+1, n)}\right|<2 \pi$. In this algorithm, we utilize traditional unwrapping method with (3) to unwrap the measured phase initially. Considering the truth that the actual phase curve will be parabola when the antenna moves along the tag, we detect the monotonicity on both sides of the extreme point. To search the extreme point, an easy way is comparing the phase differences $\Delta \psi_{m}^{(n+1, n)}, n=$ $1,2, \cdots, N-1$. Where the minimum difference is found can be regarded as the extreme point, while the index is given by $\mathrm{ID}_{\min } \leftarrow \arg \min \left|\Delta \psi_{m}^{(n+1, n)}\right|$. Otherwise, we can also find $I D_{\text {min }}$ through fitting the initial unwrapping phase curve $\psi_{m}$ and searching the minimum. Finally, we compensate the phases not satisfying the monotonicity with $\pm 2 \pi$, as shown in Algorithm 1.

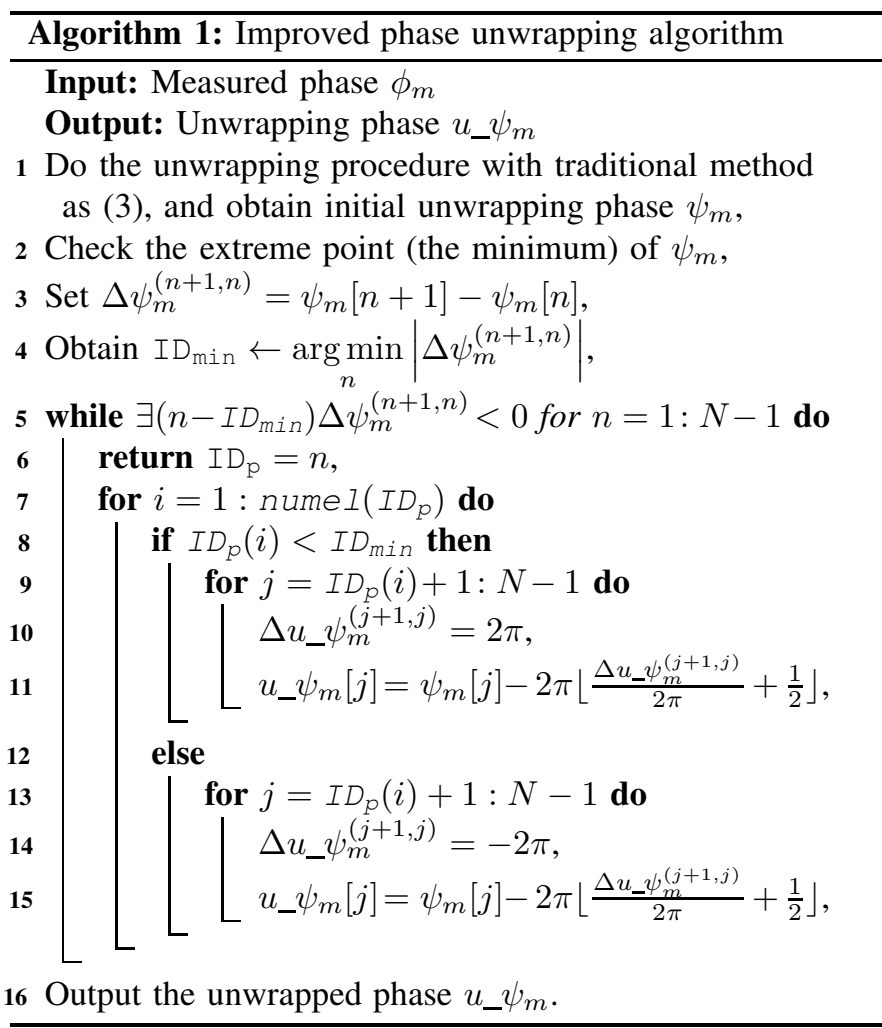

\section{B. Ranging Estimation}

According to spatial sampling theory and Remark 1, when select the first measured phase as the baseline, we obtain 


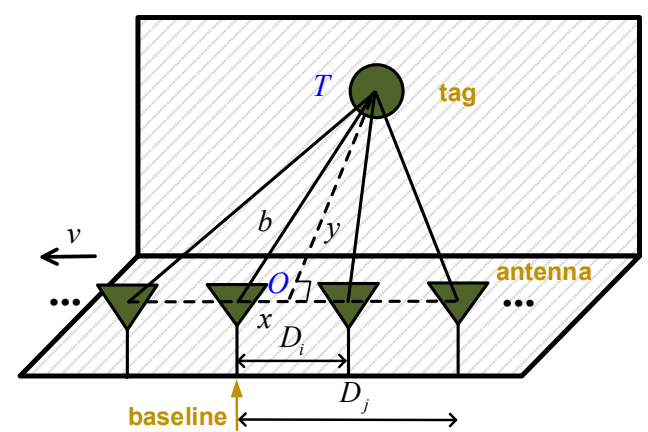

Fig. 1. Geometry of ranging estimation.

$\psi_{m}[n]+2 k_{1} \pi=4 \pi \frac{d[n]}{\lambda}+\varphi_{0}, n=1,2, \cdots, N$, so the distance differences can be calculated as

$$
\Delta d_{(i, j)}=\frac{\lambda}{4 \pi} \Delta \psi_{m}^{(i, j)},(i, j=1,2, \cdots, n, i \neq j) .
$$

So if we know the moving distances of antenna, we can calculate the distances from the antenna to the tag. But in a practical scenario, the measured phases may suffer from unexpected external interference, such as the moving human, etc. The measured phases at these positions will vary greatly and become unreliable. In this paper, we quantify the uncertainty of measured phases at $n$-th position with entropy $w[n]$,

$$
\begin{aligned}
& w[n]=1+\gamma \sum_{i=1}^{M}\left(P_{i}[n] \ln P_{i}[n]\right), \\
& P_{i}[n]=\frac{\phi_{m}^{(i)}[n]-\bar{\phi}_{m}[n]}{\sum_{j=1}^{M}\left(\phi_{m}^{(j)}[n]-\bar{\phi}_{m}[n]\right)},
\end{aligned}
$$

where $M, \bar{\phi}_{m}[n]$ are the sampling number and mean measured phase at $n$-th position, and $\gamma=\frac{1}{\ln M}$ is to normalize the entropy. When all the measured phases are very close to each other, then $P_{i}[n]$ will approach to $1 / M$, so the entropy $w[n] \rightarrow 0$, otherwise $w[n] \rightarrow 1$ when severe interference involved.

Sorting the entropy in ascending order, namely, $\mathbf{w}=$ $\left(w_{1}, w_{2}, \cdots, w_{N}\right)$. We abandon the $N-L$ measured phases with significantly large entropy, thus the first $L(L>3)$ positions with smaller entropy are selected to calculate the distances. The position with smallest entropy is chose as the baseline, so the distances from the $L-1$ positions to the baseline are redefined as $\mathbf{D}=\left(D_{1}, D_{2}, \cdots, D_{L-1}\right)$, and the corresponding distance differences from the antenna to the tag compared with the baseline are given as $\Delta \mathbf{d}=$ $\left(\Delta d_{1}, \Delta d_{2}, \cdots, \Delta d_{L-1}\right)$. Define the distance from the tag to the antenna's trajectory $|T O|=y$, and the distance from reference position to point $O$ is $x$, as shown in Fig. 1, then we obtain

$$
\sqrt{\left(x \pm D_{i}\right)^{2}+y^{2}}-\sqrt{x^{2}+y^{2}}=\Delta d_{i}, i=1,2, \cdots, L-1,
$$

where \pm represents the position left to reference position in case of ' ${ }^{\prime}$, otherwise it is ' ${ }^{\prime}$. Redefine $a=x$ and $b=$ $\sqrt{x^{2}+y^{2}}$, substitute them to (8), then we have

$$
\mathbf{A x}=\mathbf{b}
$$

where

$$
\mathbf{A}=\left(\begin{array}{cc} 
\pm 2 D_{1} & -2 \Delta d_{1} \\
\pm 2 D_{2} & -2 \Delta d_{2} \\
\vdots & \vdots \\
\pm 2 D_{L-1} & -2 \Delta d_{L-1}
\end{array}\right), \mathbf{b}=\left(\begin{array}{c}
\Delta d_{1}^{2}-D_{1}^{2} \\
\Delta d_{2}^{2}-D_{2}^{2} \\
\vdots \\
\Delta d_{L-1}^{2}-D_{L-1}^{2}
\end{array}\right)
$$

$\mathbf{x}=(a, b)^{\top}$. So we can use the least-squares method to calculate the distance from reference position to the tag, namely, $\mathbf{x}=\left(\mathbf{A}^{\top} \mathbf{A}\right)^{-1} \mathbf{A}^{\top} \mathbf{b}$, then obtain the distances at the other $L-1$ positions based on distance differences $\Delta \mathbf{d}$.

\section{Inter-tag Positioning}

Based on (6) and (9), we can obtain the distances from antenna to tag when antenna moving along the tag. Instead of obtaining the absolute positions of the tags, we focus on the inter-tag relative locations. Fig. 2 presents the case that the antenna at two positions, and we project the two positions onto the plane of the rack. To obtain inter-tag relative locations, we choose one of the tags as the initiation tag (marked as $R$ in Fig. 2), which should not be collinear with the two projected antenna positions (marked as $A_{1}$ and $A_{2}$ ). It can be noted that there are two cases for the unknown tag (marked as $U$ ) when considering the positional relationship between $U$ and triangle $\Delta R A_{1} A_{2}$ : outside and inside (including locating on the sides), as shown in Fig. 2.

To know the relative position towards the reference tag, we need to calculate the distance $\iota_{r u}$ to the unknown tag and the rotation angle $\vartheta$, where $\vartheta$ is defined as the angle rotation from left axis of reference tag to the connecting line between the unknown tag and reference tag (while the clockwise rotation is negative, otherwise, it is positive). Moreover, when the previous selected reference tag is out of antenna's working distance, one of the tags has been positioned previously can be chose as the new reference tag. So the relative angle $\vartheta$ can be defined as $\vartheta=\theta_{1} \pm \theta_{2}$. When the unknown tag locates outside the triangle $\Delta R A_{1} A_{2},{ }^{\prime} \pm^{\prime}$ will take ${ }^{\prime}+{ }^{\prime}$ in case of $\theta_{1}>\theta_{3}$, and ' $-{ }^{\prime}$ in case of $\theta_{1}<\theta_{3}$, where $\theta_{1}, \theta_{2}$ and $\theta_{3}$ is one of the interior angles of the triangles $\Delta R A_{1} A_{2}$ and

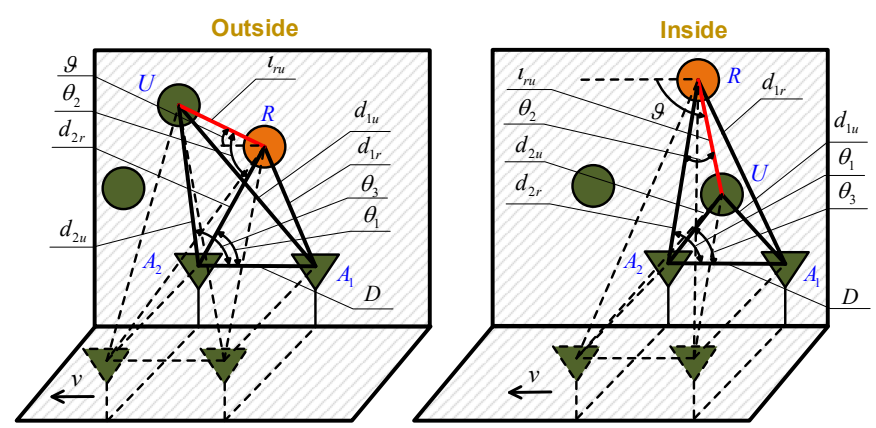

Fig. 2. Geometry diagram of relative positioning. 
$\triangle R U A_{2}$, respectively. When the unknown tag is in the triangle $\Delta R A_{1} A_{2},{ }^{\prime} \pm^{\prime}$ will take ${ }^{\prime}+{ }^{\prime}$. To sum up, ${ }^{\prime}+{ }^{\prime}$ represents the case of $\theta_{1}>\theta_{3},{ }^{\prime}-^{\prime}$ is for the case that $\theta_{1}<\theta_{3}$. Due to $\theta_{1}, \theta_{2} \in(0, \pi)$, the relative angle $\vartheta \in(-\pi, \pi)$. Based on the Law of Cosines, we can obtain the relative distance $\iota_{r u}$, the involved angles $\theta_{1}, \theta_{2}$, and $\theta_{3}$,

$$
\begin{aligned}
\cos \theta_{1} & =\frac{d_{2 r}^{2}+D^{2}-d_{1 r}^{2}}{2 d_{2 r} D}, \\
\cos \theta_{3} & =\frac{d_{2 u}^{2}+D^{2}-d_{1 u}^{2}}{2 d_{2 u} D}, \\
\iota_{r u}^{2} & =d_{2 u}^{2}+d_{2 r}^{2}-2 d_{2 u} d_{2 r} \cos \left(\theta_{3}-\theta_{1}\right), \\
\cos \theta_{2} & =\frac{\iota_{r u}^{2}+d_{2 r}^{2}-d_{2 u}^{2}}{2 \iota_{r u} d_{2 r}},
\end{aligned}
$$

where the parameters of angle and distance involved are given in Fig. 2.

\section{EXPERIMENT AND RESULTS}

\section{A. Configuration}

To validate our method, the RePos RFID positioning system has been established, as shown in Fig. 3. The Impinj Speedway R420 RFID reader [10] is used without any hardware modification, which supports four directional antennas at most. The reader is connected to PC controller (Dell laptop E7450, which equips Inter(R) Core(TM) i7-5600U CPU $@ 2.60 \mathrm{GHz}$ and $16 \mathrm{~GB}$ RAM) through the Ethernet cable under the LLRP protocol. The UHF-RFID Antenna is a Keonn Advantenna-SP11, which is a compact antenna with circular polarization, and the dimensions are $207 \times 207 \times 11.6$ mm. Seven DogBone tags with the Impinj Monza 4D chip are employed. In the experiment, six tags are adhered on the boxes on the rack (the scale is $1.2 \times 2 \mathrm{~m}$ ), while one tag is on the plastic board, as shown in Fig. 3. The Impinj R420 reader mode is set 'Auto Dense Set', and search mode is set 'Single Target', and the transmitted power is $18 \mathrm{dBm}$.

\section{B. Method Performance}

In the first experiment, the antenna moves along the rack linearly, and the distance to the rack is $0.9 \mathrm{~m}$. The antenna's moving step is $0.1 \mathrm{~m}$, which satisfies the spatial sampling

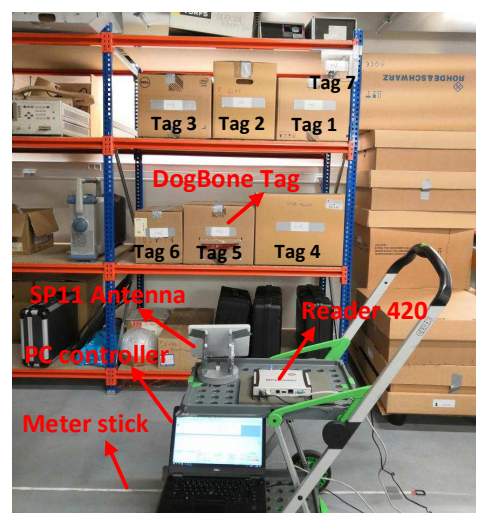

Fig. 3. UHF-RFID positioning system: experiment setups.

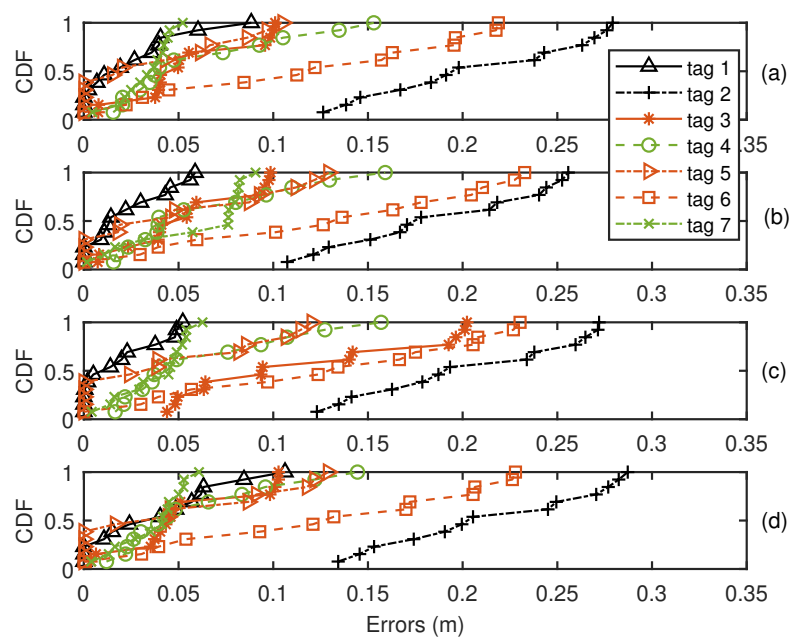

Fig. 4. CDF of ranging errors: (a) channel: $865.7 \mathrm{MHz}$, (b) channel: 866.3 MHz, (c) channel: $866.9 \mathrm{MHz}$, (d) channel: $867.5 \mathrm{MHz}$.

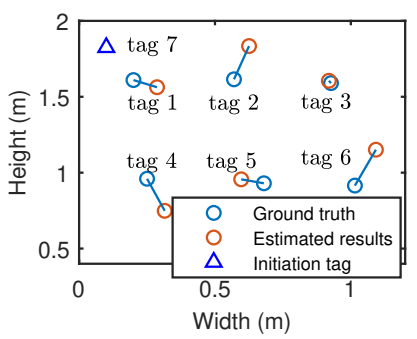

(a) Channel: $865.7 \mathrm{MHz}$

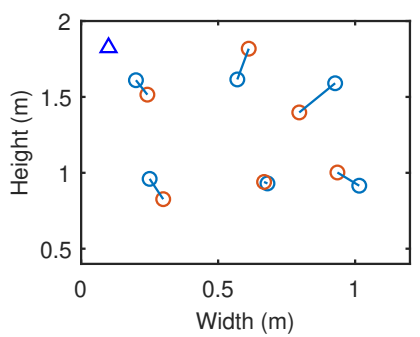

(c) Channel: $866.9 \mathrm{MHz}$

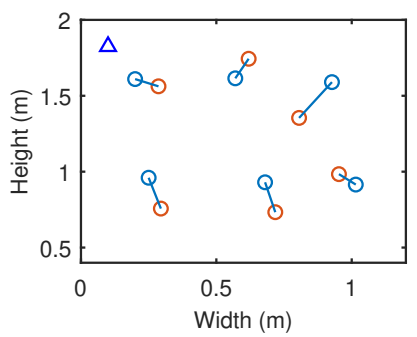

(b) Channel: $866.3 \mathrm{MHz}$

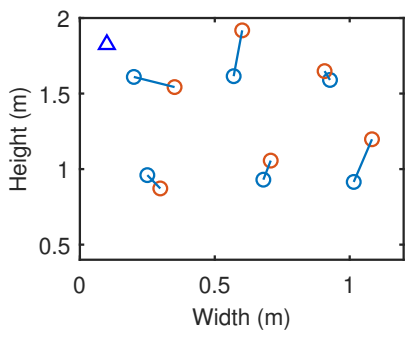

(d) Channel: $867.5 \mathrm{MHz}$
Fig. 5. The positioning results of four channels with the initiation tag 7 .

theory, even through it is a little larger than quarter wavelength (about $0.086 \mathrm{~m}$ for frequency band $865-868 \mathrm{MHz}$ ). In this case, we can obtain the unwrapping phases without further compensation using $\pm 2 \pi$. Fig. 4 compares the ranging errors under four channels (865.7 MHz, 866.3 MHz, 866.9 MHz and $867.5 \mathrm{MHz}$ [11]), the frequency interval is $0.6 \mathrm{MHz}$. The cumulative distribution function (CDF) is used to describe the ranging estimation performance of the proposed algorithm. It can be seen that seven tags' positioning errors present a similar error distribution under different channels, namely there is no distinct frequency selectivity in this case. For tag 1 and tag 7, the ranging errors are less than $0.11 \mathrm{~m}$, while tag 2 has the largest errors $(0.28 \mathrm{~m})$ among the seven tags. So our method can realize 0.28 -meter ranging accuracy in general. 


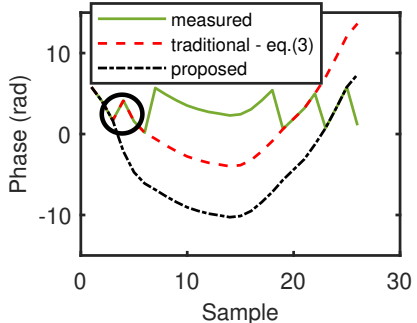

(a) Measured phase unwrapping

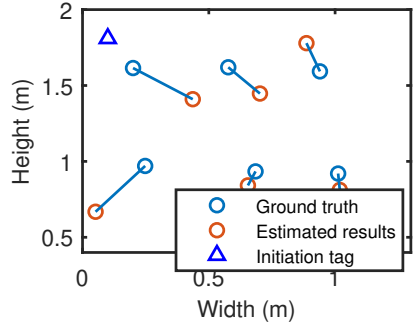

(b) Positioning results
Fig. 6. The positioning results using improved phase unwrapping.

In the experiment, we select tag 7 on the top right as the initiation tag to start relative locations planning. The antenna moves along the rack from the right side to the left side, so tag 7 will be read at the early stage when the seven tags enter the interrogation region of the antenna. It should be noted that tag 7 is not a reference tag, and the exact position is not required in practical applications. We can still obtain the relative positions of the tags based on the inter-tag distance and angles, even though the position of initiation tag is given arbitrarily. The results of the relative inter-tag positions are presented in Fig. 5. To show the positioning accuracy visually, we give the ground truth of initiation tag and other six tags. As we can see from Fig. 5, besides ranging accuracy, the positioning accuracy also greatly depends on the relative angle estimation. Specifically, for tag 2, the estimation errors in Fig. 5(a)(d) are mainly caused by the angle errors, while the largest angle errors reach 0.4 rad. Generally, according to the positioning results and the ground truth in Fig. 5, the proposed method can distinguish the tags on each level and column without ambiguity.

In the second experiment, we increase the distance from the antenna's trajectory to the rack to $1.1 \mathrm{~m}$, and keep the moving step as $0.1 \mathrm{~m}$. In this case, we observe that not all the tag (such as tag 2 on the rack) can use (3) to unwrap the measured phases even though they still satisfy the spatial sampling theory according to our geometry calculation, which may come from unintended measured errors or collisions. In Fig. 6(a), it presents the original measured phases of tag 2 with the frequency $866.9 \mathrm{MHz}$, unwrapping results using (3) and the proposed method (Algorithm 1), respectively. As shown by the black ellipse in Fig. 6(a), unwrapping method using (3) in this case fails to unwrap one sample, but the phases after this sample are also totally wrong, which can not be used to conduct range estimation. As for the improved method, it can unwrap the measured phases successfully. Fig. 6(b) presents the positioning results using the unwrapping phases. In this case, our method is still able to distinguish the tag-sticking items successfully.

\section{CONCLUSION}

This paper proposed a relative positioning method for UHF-RFID passive tag. Instead of absolute coordinates, the proposed method focuses on the relative locations' relationship among the tags through calculating the inter-tag distances and angles. To unwrap the measured phases, the improved unwrapping method has been proposed on the basis of spatial sampling theory with relaxing constraint. Moreover, considering the unintended mobile object's interference, the reliable measured phases have been selected to estimate the distance based on the entropy at each position. According to experimental results, the proposed method can realize 0.28 meter lever ranging accuracy generally, and distinguish the tags around horizontally and vertically without ambiguity. A main future work will consist of investigating the potential of positioning the tags at different vertical planes, and improving the robustness to the location errors of antenna (reader).

\section{ACKNOWLEDGMENT}

This work is supported in part by the Excellence of Science (EOS) project MUlti-SErvice WIreless NETworks (MUSEWINET), and IMEC co-financed project InWareDrones.

\section{REFERENCES}

[1] G. WANG, C. Qian, L. Shangguan, H. Ding, J. Han, K. Cui, W. Xi, and J. Zhao, "Hmo: Ordering rfid tags with static devices in mobile environments," IEEE Transactions on Mobile Computing, pp. 1-1, 2019.

[2] L. Yang, Y. Chen, X.-Y. Li, C. Xiao, M. Li, and Y. Liu, "Tagoram: Realtime tracking of mobile rfid tags to high precision using cots devices," in Proceedings of the 20th Annual International Conference on Mobile Computing and Networking, ser. MobiCom '14. New York, NY, USA: ACM, Sep 2014, pp. 237-248.

[3] A. Buffi, P. Nepa, and F. Lombardini, "A phase-based technique for localization of uhf-rfid tags moving on a conveyor belt: Performance analysis and test-case measurements," IEEE Sensors Journal, vol. 15, no. 1, pp. 387-396, Jan 2015.

[4] A. Buffi and P. Nepa, "The sarfid technique for discriminating tagged items moving through a uhf-rfid gate," IEEE Sensors Journal, vol. 17, no. 9, pp. 2863-2870, May 2017.

[5] A. Buffi, A. Motroni, P. Nepa, B. Tellini, and R. Cioni, "A sar-based measurement method for passive-tag positioning with a flying uhfrfid reader," IEEE Transactions on Instrumentation and Measurement, vol. 68, no. 3, pp. 845-853, Mar 2019.

[6] L. Shangguan, Z. Li, Z. Yang, M. Li, and Y. Liu, "Otrack: Order tracking for luggage in mobile rfid systems," in 2013 Proceedings IEEE INFOCOM, Apr 2013, pp. 3066-3074.

[7] L. Shangguan, Z. Yang, A. X. Liu, Z. Zhou, and Y. Liu, "Relative localization of RFID tags using spatial-temporal phase profiling," 12th USENIX Symposium on Networked Systems Design and Implementation (NSDI 15), pp. 251-263, May 2015.

[8] L. Shangguan and Kyle Jamieson, "The Design and Implementation of a Mobile RFID Tag Sorting Robot,” MobiSys, pp. 31-42, Jun 2016.

[9] H. Wu, B. Tao, Z. Gong, Z. Yin, and H. Ding, "A fast uhf rfid localization method using unwrapped phase-position model," IEEE Transactions on Automation Science and Engineering, pp. 1-10, 2019.

[10] Impinj, "Speedway revolution reader application note - low level user data support (revision 3.0, 2013)," [Online]. Available: https://support. impinj.com/hc/en-us/articleattachments.

[11] EPC Radio-Frequency Identity Protocols Generation-2 UHF RFID, "Specification for rfid air interface protocol for communications at 860 MHz - 960 MHz,” 2015. 